\title{
Conocimientos, habilidades y actitudes profesionales para la intervención breve en adicciones
}

\author{
Silvia Morales-Chainé', Violeta Félix Romero', Germán Palafox Palafox', José Luis Vázquez Martínez² \\ I Universidad Nacional Autónoma de México \\ 2 Unidad de Reducción de la Demanda de la Comisión Interamericana para el Control del Abuso de Drogas
}

\section{RESUMEN}

Introducción: la capacitación continua y certificación de los profesionales de atención primaria de salud apoyan la implementación de intervenciones breves para abordar el uso de drogas. Objetivo: validar los materiales de capacitación del "Curriculum Universal de Tratamiento para Profesionales de la Salud" mediante la evaluación de los conocimientos, habilidades y actitudes. Método: se trabajó con 823 profesionales (137 de Argentina, 59 de Colombia, 86 de El Salvador, 398 de México y 143 de Uruguay), en cuanto a la utilización de la intervención breve, según la modalidad de capacitación en la que participaron (272 presencial, 125 mixta y 426 a distancia). Se aplicó: evaluación escrita de conocimientos, evaluación conductual de habilidades-actitudes y un cuestionario de satisfacción. Se utilizó un diseño cuasi-experimental post, con grupos de comparación para evaluar los conocimientos, habilidades, actitudes (a través de observación directa) y satisfacción de los profesionales de la salud. Resultados: los de la evaluación escrita y observación directa indican que, tanto los materiales de capacitación como la estrategia pedagógica empleada, incrementaron conocimientos, habilidades y actitudes para la implementación de la intervención breve. Conclusiones: se obtuvo una herramienta de diseminación exitosa, basada en los contenidos recomendados por organismos internacionales y validada en el campo de aplicación clínica de los principios básicos del comportamiento.

Palabras clave: intervención breve, adicciones, capacitación a distancia, atención primaria.

\begin{abstract}
Introduction: the continuous training and certification of primary healthcare professionals support the implementation of brief interventions to address drug use. Objective: our objective was to validate the training materials of the "Universal Treatment Curriculum for Health Professionals" by assessing the knowledge, skills, and attitudes. Method: we worked with 823 health professionals (137 from Argentina, 59 from Colombia, 86 from El Salvador, 398 from Mexico and 143 from Uruguay), regarding the utilization of brief intervention, according to the training modality in which they participated (272 face-to-face, 125 mixed, and 426 online). We applied a written knowledge evaluation, behavioral assessment of skills - attitudes, and a Satisfaction Questionnaire. A post-quasi-experimental design was used, with comparison groups to assess knowledge, skills, attitudes (through direct observation) and satisfaction of health professionals. Results: the results of the written evaluation and direct observation indicate that both, the training materials and the pedagogical strategy, increased knowledge, skills, and attitudes for the implementation of brief interventions. Conclusions: in this way, a successful dissemination tool was obtained, based on the contents recommended by international organizations and validated in the field of clinical application of the basic principles of behavior.
\end{abstract}

Keywords: Brief intervention, addictions, distance training, primary care.

\section{Autor de correspondencia:}

Silvia Morales-Chainé. Correo electrónico: smchaine@gmail.com Recibido: 12 de agosto de 2019 Aceptado: 8 de octubre de 2019 doi: 10.28931/riiad.2019.2.02 


\section{INTRODUCCIÓN}

El consumo de drogas es un fenómeno mundial, con implicaciones y costos políticos, económicos, sociales y ambientales. Es complejo, dinámico, multicausal y representa un desafío a las naciones que requiere abordarse integralmente, mediante un enfoque multidisciplinario, como una responsabilidad común y compartida entre los países (Estrategia Hemisférica sobre Drogas, 2010). En el panorama mundial están presentes tanto el consumo de drogas, como la necesidad aún insatisfecha de atender a las diversas poblaciones con problemas derivados de ello. El objetivo principal de cualquier política de reducción de la demanda de drogas no solamente consiste en prevenir y tratar los problemas por consumo de drogas; de manera más amplia, se busca reducir o minimizar las consecuencias sociales (Organización de los Estados Americanos, OEA, 2013).

De acuerdo con el Informe Mundial de Drogas (2018), en el año 2016 se registró que 275 millones de personas refirieron haber consumido drogas alguna vez en la vida; de los cuales 31 millones presentaron trastornos por consumo de drogas. En los países de las Américas existen grandes variaciones y destaca el alto consumo en adolescentes con baja percepción del riesgo. Además, se presentan retos importantes como el aumento en el uso de opiáceos y nuevas formas de abuso de drogas que se han consumido desde hace varios años.

La transición de los primeros contactos experimentales con las drogas hacia la dependencia se asocia con una variedad de factores de riesgo relacionados con el individuo y su ambiente (Informe Mundial de Drogas, 2018). Generalmente se describe un patrón evolutivo que comienza con una actividad voluntaria de consumo. Al inicio, prevalece el efecto positivo y placentero que la persona que consume reporta que controla. De manera paulatina, en algunas personas la motivación va cambiando y pasan de consumir para experimentar placer a hacerlo para evitar el malestar, para poder estar o sentirse bien, aumentando la dosis y la frecuencia (Comisión Interamericana para el Control del Abuso de Drogas - Organización de los Estados Americanos, ClCAD-OEA, 2017). Finalmente, algunas personas pierden el control sobre el uso y reportan incapacidad para suspenderlo a pesar de conocer los riesgos y daños.

Existen intervenciones que consideran las primeras etapas en la evolución y desarrollo del trastorno. En 2004, la Organización Mundial de la Salud (OMS) recomendó la aplicación de intervenciones breves para prevenir problemas de salud y reducir el daño ocasionado por los mismos. En estas intervenciones, se diseña un plan específico para cada problema a partir de identificar la situación a solucionar, establecer metas, plantear intervenciones para lograr esas metas, examinar las respuestas para corregir su enfoque y, finalmente, evaluar el resultado de la intervención respecto a su eficacia (Zepeda, Ávila, Morales-Chainé, \& Martín del Campo, 2017). Para esta modalidad de intervención, la formulación diagnóstica corresponde a la versión más simple de la evolución y el mantenimiento de una problemática específica, por lo cual se busca intervenir de manera rápida a partir de la exploración y elaboración sostenida de conductas o actitudes que tienden a maximizar la resolución efectiva de los problemas. De manera concreta, la intervención breve es pragmática y se centra en el análisis de las ideas (constructos) y secuencias conductuales repetitivas que rodean al trastorno. Sus principales objetivos son modificar creencias o constructos relacionados con la problemática, así como secuencias repetitivas relacionadas con la problemática (Rodríguez, Echeverría, Martínez, \& Morales-Chainé, 2017).

Ante este panorama, a través del consenso, en los países de las Américas se ha planteado una respuesta estratégica con un componente de capacitación continua y certificación del talento humano que otorga servicios a las personas con problemas por consumo de drogas, donde destacan las intervenciones breves (Plan de Acción Hemisférico sobre Drogas, 2016-2020). Para esto es fundamental aplicar programas de capacitación continua, basados en competencias, en colaboración con instituciones académicas y especializadas como las universidades (Atlas de Salud Mental, 2014).

En el ámbito de la capacitación para el manejo de las intervenciones breves en adicciones, en México, Morales-Chainé, Martínez, Carrazcosa, Chaparro, y Martínez (2013) evaluaron las habilidades de 133 profesionales y 279 no profesionales de la salud (promedio de 32 años de edad, $77 \%$ mujeres, $50 \%$ con un nivel mínimo de licenciatura) con situaciones simuladas de interacción promotor-persona que consume, a través de un diseño factorial de dos por cuatro: profesionales versus no profesionales en una de cuatro condiciones: a) entrenamiento con material, b) entrenamiento sin material, c) con material sin entrenamiento y d) $\sin$ material y sin entrenamiento. El entrenamiento y material se constituyeron alrededor de las habilidades de intervención breve: identifica, dialoga, establece metas y acompaña. Los resultados mostraron que la puntuación promedio y por escala, específicamente de las habilidades para indagar, dialogar y establecer metas, fue mayor para los profesionales de la salud en cualquiera de las tres condiciones de entrenamiento. Los autores concluyeron que tanto el entrenamiento presencial en habilidades como el material son efectivos para aprender las habilidades de la intervención breve para la atención de las adicciones. 
Posteriormente, también en México, Morales-Chainé, Robles, Nieto y Martín del Campo (2016) describieron el proceso de diseminación de las intervenciones breves para la prevención y atención del consumo de drogas a través de un programa de capacitación a distancia para profesionales de la salud en colaboración con la Universidad Nacional Autónoma de México (UNAM). La población blanco la conformaron 2,940 profesionales de la salud de unidades de atención primaria a las adicciones, $76 \%$ psicólogos, $7 \%$ médicos y 17\% trabajadores sociales. En el programa se emplearon técnicas y estrategias de educación a distancia, producto de una necesidad para dar respuesta a la difusión de las innovaciones tecnológicas y de actualización sostenida del conocimiento. Los autores reportaron que 1,040 profesionales acreditaron un diplomado de 240 horas, 799 concluyeron la especialización a distancia (816 horas) y 1,101 acreditaron módulos por constancia (en un rango de 32 a 80 horas). De ahí se reportó la aplicación de 656,111 pruebas de detección oportuna y la aplicación de intervenciones breves a 68,798 adolescentes. Se concluyó que la formación de profesionales de la salud (medicina, psicología y trabajo social) de las unidades de atención primaria del país abona a la implementación de la prevención y atención de las adicciones como un esfuerzo coordinado entre las diversas instancias, comprometidas en disminuir el daño provocado por el inicio temprano y el consumo de drogas en la población. Los autores también sugirieron la necesidad de ilustrar el nivel de conocimientos teóricos y prácticos adquiridos, el efecto de la capacitación de profesionales de la salud y el aporte práctico de las tecnologías de la información en el entrenamiento supervisado para la atención de las adicciones.

En el 2012, Vargas, Martínez, Pedroza y Morales-Chainé evaluaron los materiales de capacitación a distancia de un modelo de intervención breve para adolescentes que se inician en el consumo del alcohol. La plataforma Moodle $®$ contenía foros de discusión, chat, un audio, 24 videos de modelamiento por profesionales con más de cinco años de experiencia en el campo de las adicciones, folletos, imágenes, bitácoras de trabajo y actividades de aprendizaje para la capacitación del modelo de intervención breve a lo largo de cinco semanas, en la que colaboraron 20 profesionales de la psicología de 34 años de edad, en promedio, que hicieron observaciones acerca de la pertinencia de contenidos, instrucciones y actividades de aprendizaje, y evaluaron la usabilidad de la plataforma. Los autores concluyeron que la capacitación a distancia permitió incrementar las habilidades para el manejo del internet, mejorar el funcionamiento de la plataforma, los conocimientos sobre uso y abuso de drogas y el programa de intervención breve para ado- lescentes. También se reportó una satisfacción mayor al $70 \%$ en todos los participantes.

Recientemente, Barrera, Echeverría y Morales-Chainé (2017) Ilevaron a cabo un estudio con 136 profesionales de la salud a quienes capacitaron a través de una plataforma Moodle ${ }^{\circledR}$ sobre las estrategias de intervención breve para abordar consumo excesivo de alcohol. Del total de participantes, $77 \%$ fueron mujeres de 33 años de edad, en promedio, y $85 \%$ profesionales de psicología, con licenciatura como escolaridad mínima. Se supervisó a cada profesional en la atención de una persona que consumía alcohol, hasta concluir la intervención breve. Los autores reportaron que a partir de la capacitación, las personas que consumían alcohol decrementaron su consumo de 11 bebidas estándar en promedio a una bebida estándar en la cuarta sesión de intervención breve y hasta el seguimiento a los seis meses, y registraron incremento en su autoeficacia. Más del $90 \%$ de profesionales calificaron los materiales como adecuados en la información, el enfoque teórico, la intervención breve, suficientes ejemplos, claridad en las explicaciones y que comprendieron adecuadamente los contenidos; que consideraban la educación a distancia adecuada para aprender y de buena calidad.

Sin embargo, y en contraste con lo que plantea el consenso político y la experiencia previa, la realidad actual de las Américas muestra que el financiamiento y las capacidades de atención de adicciones son claramente insuficientes. Sobresalen grandes retos en cuanto a profesionales (médicos, enfermeras, trabajadores sociales, psicólogos, etcétera) capacitados e integración de servicios para llevar a cabo intervenciones breves para prevención, tratamiento y reinserción social en el ámbito de las adicciones; pero también es ahí donde existen grandes áreas de oportunidad para la formación expedita y concreta.

Para abordar la situación, desde CICAD se han desarrollado y apoyado iniciativas de capacitación, actualización y certificación del talento humano que presta servicios en los países, como el Currículum Universal de Prevención (UPC) y de tratamiento (UTC; CICAD, 2017). Con base en estas experiencias, se analizó y acordó cuáles son las funciones básicas y competencias que los profesionales de la salud deben tener en cribado e intervención temprana, tratamiento, monitoreo y seguimiento. Derivado de ello, CICAD y UNAM desarrollaron los materiales del Currículum Universal de Tratamiento de adicciones (UTC, por sus siglas en inglés) para profesionales de la salud, con un enfoque pedagógico monitoreado por la Facultad de Psicología de la UNAM, con la intención de capacitar de manera efectiva, a corto plazo, en el uso de intervenciones breves basadas en evidencia científica, a través de un curso que permitiera 
obtener competencias (conocimientos, habilidades y actitudes) de desempeño clínico.

Por lo tanto, el objetivo del presente trabajo fue evaluar el nivel de conocimientos, habilidades y actitudes de profesionales de la salud para la intervención breve en adicciones, en función de la modalidad de capacitación: presencial, mixta y a distancia; como estrategia de validación de los materiales didácticos del UTC para profesionales de la salud.

\section{MÉTODO}

\section{Participantes}

Se trabajó con 823 profesionales de la salud: 137 de Argentina, 59 de Colombia, 86 de El Salvador, 398 de México y 143 de Uruguay (ver Tabla 1) en tres modalidades de capacitación: a) presencial, con 272 personas (78\% mujeres, con edad promedio de 37 años); b) mixta, con 125 personas (78\% mujeres, con edad promedio de 36 años), y c) a distancia, con 426 personas (77\% mujeres, con edad promedio de 34 años). Los participantes fueron invitados, por lo que los grupos de la investigación no pudieron ser homogéneos en cantidad o en nivel académico. Los perfiles profesionales corresponden a 488 de psicología, 74 de medicina, 107 de trabajo social, 55 de enfermería, 10 de psiquiatría y 89 de otras profesiones (p. ej., contaduría y administración o docentes).

Los profesionales firmaron un consentimiento informado en el que se estableció la duración de su participación, que los resultados del estudio se usarían para investigación epidemiológica y difusión de resultados; que se mantendría la confidencialidad de la información, a través de la utilización de promedios grupales; y que tenían derecho a declinar el uso de su información y participación en cualquier momento del estudio, sin perjudi-

Tabla 1

Número de participantes en función del país de origen, de la modalidad de capacitación, del perfil profesional y el género

\begin{tabular}{|c|c|c|c|c|c|c|c|c|c|c|c|c|c|c|c|}
\hline \multirow{3}{*}{ País } & \multirow{3}{*}{ Modalidad } & \multicolumn{14}{|c|}{ Profesión/Sexo } \\
\hline & & \multicolumn{2}{|c|}{ Psicología } & \multicolumn{2}{|c|}{ Medicina } & \multicolumn{2}{|c|}{ Trabajo Social } & \multicolumn{2}{|c|}{ Enfermería } & \multicolumn{2}{|c|}{ Psiquiatría } & \multicolumn{2}{|c|}{ Otro } & \multicolumn{2}{|c|}{ TOTAL } \\
\hline & & M & $\mathrm{H}$ & $\mathrm{M}$ & $\mathrm{H}$ & $\mathrm{M}$ & $\mathrm{H}$ & $M$ & $\mathrm{H}$ & $\mathrm{M}$ & $\mathrm{H}$ & $\mathrm{M}$ & $\mathrm{H}$ & $\mathrm{M}$ & $\mathrm{H}$ \\
\hline \multicolumn{16}{|c|}{ Argentina } \\
\hline \multirow{2}{*}{\multicolumn{2}{|c|}{ Presencial }} & 16 & 1 & 4 & 1 & 1 & 0 & 1 & 1 & 0 & 0 & 2 & 2 & 24 & 5 \\
\hline & & \multicolumn{2}{|c|}{17} & \multicolumn{2}{|c|}{5} & \multicolumn{2}{|c|}{1} & \multicolumn{2}{|c|}{2} & & & \multicolumn{2}{|c|}{4} & \multicolumn{2}{|c|}{29} \\
\hline \multirow{2}{*}{\multicolumn{2}{|c|}{ Mixto }} & 3 & 1 & 2 & 0 & 0 & 0 & 0 & 0 & 0 & 0 & 0 & 0 & 5 & 1 \\
\hline & & \multicolumn{2}{|c|}{4} & \multicolumn{2}{|c|}{2} & \multicolumn{2}{|c|}{0} & \multicolumn{2}{|c|}{0} & \multicolumn{2}{|c|}{0} & \multicolumn{2}{|c|}{0} & \multicolumn{2}{|c|}{6} \\
\hline \multirow{2}{*}{\multicolumn{2}{|c|}{ A distancia }} & 69 & 8 & 1 & 1 & 3 & 0 & 2 & 1 & 1 & 1 & 13 & 2 & 89 & 13 \\
\hline & & \multicolumn{2}{|c|}{77} & \multicolumn{2}{|c|}{2} & \multicolumn{2}{|c|}{3} & \multicolumn{2}{|c|}{3} & \multicolumn{2}{|c|}{2} & \multicolumn{2}{|c|}{15} & \multicolumn{2}{|c|}{102} \\
\hline \multirow{2}{*}{\multicolumn{2}{|c|}{ Total }} & 88 & 10 & 7 & 2 & 4 & 0 & 3 & 2 & 1 & 1 & 15 & 4 & 118 & 19 \\
\hline & & & & & & & & & & & & & & & \\
\hline
\end{tabular}

Colombia

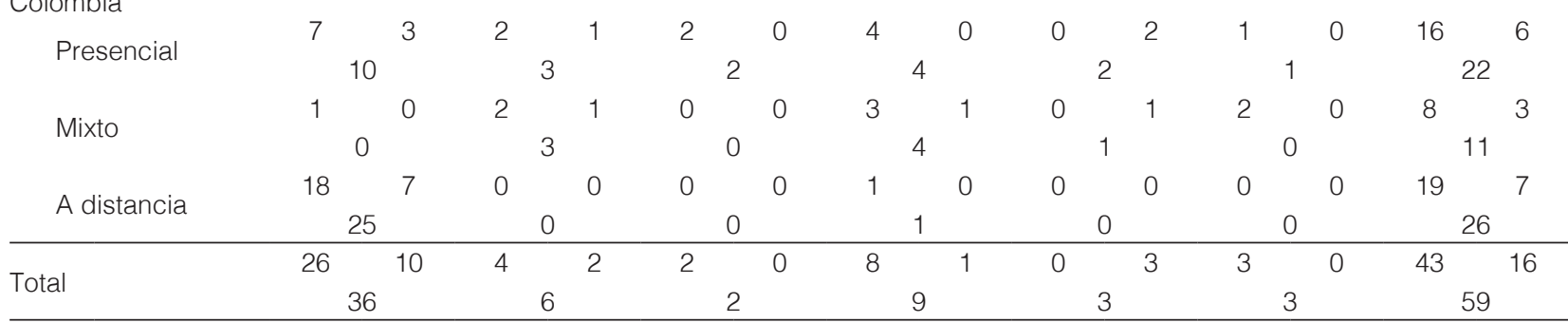

El Salvador

\begin{tabular}{|c|c|c|c|c|c|c|c|c|c|c|c|c|c|c|}
\hline \multirow{2}{*}{ Presencial } & 11 & 0 & 8 & 6 & 2 & 0 & 8 & 1 & 2 & 1 & 0 & 0 & 31 & 8 \\
\hline & \multicolumn{2}{|c|}{11} & \multicolumn{2}{|c|}{14} & \multicolumn{2}{|c|}{2} & \multicolumn{2}{|c|}{9} & \multicolumn{2}{|c|}{3} & \multicolumn{2}{|c|}{0} & \multicolumn{2}{|c|}{39} \\
\hline \multirow{2}{*}{ Mixto } & 9 & 3 & 4 & 4 & 1 & 0 & 2 & 0 & 0 & 0 & 0 & 0 & 16 & 7 \\
\hline & & & \multicolumn{2}{|c|}{8} & \multicolumn{2}{|c|}{1} & \multicolumn{2}{|c|}{2} & \multicolumn{2}{|c|}{0} & \multicolumn{2}{|c|}{0} & \multicolumn{2}{|c|}{23} \\
\hline \multirow{2}{*}{ A distancia } & 6 & 3 & 3 & 3 & 1 & 0 & 5 & 2 & 1 & 0 & 0 & 0 & 16 & 8 \\
\hline & & & \multicolumn{2}{|c|}{6} & \multicolumn{2}{|c|}{0} & \multicolumn{2}{|c|}{7} & \multicolumn{2}{|c|}{1} & \multicolumn{2}{|c|}{0} & \multicolumn{2}{|c|}{24} \\
\hline \multirow{2}{*}{ Total } & 26 & 6 & 15 & 13 & 4 & 0 & 15 & 3 & 3 & 1 & 0 & 0 & 63 & 23 \\
\hline & \multicolumn{2}{|c|}{32} & \multicolumn{2}{|c|}{28} & \multicolumn{2}{|c|}{4} & \multicolumn{2}{|c|}{18} & \multicolumn{2}{|c|}{4} & \multicolumn{2}{|c|}{0} & \multicolumn{2}{|c|}{86} \\
\hline
\end{tabular}


Tabla 1. Continúa

\begin{tabular}{|c|c|c|c|c|c|c|c|c|c|c|c|c|c|c|}
\hline \multirow{3}{*}{ Modalidad } & \multicolumn{14}{|c|}{ Profesión/Sexo } \\
\hline & \multicolumn{2}{|c|}{ Psicología } & \multicolumn{2}{|c|}{ Medicina } & \multicolumn{2}{|c|}{ Trabajo Social } & \multicolumn{2}{|c|}{ Enfermería } & \multicolumn{2}{|c|}{ Psiquiatría } & \multicolumn{2}{|c|}{ Otro } & \multicolumn{2}{|c|}{ TOTAL } \\
\hline & $\mathrm{M}$ & $\mathrm{H}$ & $\mathrm{M}$ & $\mathrm{H}$ & $\mathrm{M}$ & $\mathrm{H}$ & M & $\mathrm{H}$ & M & $\mathrm{H}$ & M & $\mathrm{H}$ & M & $\mathrm{H}$ \\
\hline \multicolumn{15}{|l|}{ México } \\
\hline \multirow{2}{*}{ Presencial } & 85 & 25 & 1 & 5 & 32 & 2 & 1 & 2 & 0 & 0 & 5 & 2 & 124 & 36 \\
\hline & \multicolumn{2}{|c|}{110} & \multicolumn{2}{|c|}{6} & \multicolumn{2}{|c|}{34} & \multicolumn{2}{|c|}{3} & \multicolumn{2}{|c|}{0} & \multicolumn{2}{|c|}{7} & \multicolumn{2}{|c|}{160} \\
\hline \multirow{2}{*}{ Mixto } & 41 & 11 & 1 & 2 & 9 & 1 & 0 & 0 & 0 & 0 & 6 & 3 & 57 & 17 \\
\hline & \multicolumn{2}{|c|}{52} & \multicolumn{2}{|c|}{3} & \multicolumn{2}{|c|}{10} & \multicolumn{2}{|c|}{0} & \multicolumn{2}{|c|}{0} & \multicolumn{2}{|c|}{9} & \multicolumn{2}{|c|}{74} \\
\hline \multirow{2}{*}{ A distancia } & 57 & 19 & 5 & 10 & 31 & 2 & 8 & 1 & 1 & 0 & 19 & 11 & 121 & 43 \\
\hline & \multicolumn{2}{|c|}{76} & \multicolumn{2}{|c|}{15} & \multicolumn{2}{|c|}{33} & \multicolumn{2}{|c|}{9} & \multicolumn{2}{|c|}{1} & \multicolumn{2}{|c|}{30} & \multicolumn{2}{|c|}{164} \\
\hline \multirow{2}{*}{ Total } & 183 & 55 & 7 & 17 & 72 & 5 & 9 & 3 & 1 & 0 & 30 & 16 & 302 & 96 \\
\hline & \multicolumn{2}{|c|}{238} & & & & & & & & & & & & \\
\hline
\end{tabular}

\begin{tabular}{|c|c|c|c|c|c|c|c|c|c|c|c|c|c|c|}
\hline \multicolumn{15}{|l|}{ Uruguay } \\
\hline \multirow{2}{*}{ Presencial } & 11 & 3 & 0 & 1 & 3 & 1 & 0 & 0 & 0 & 0 & 3 & 0 & 17 & 5 \\
\hline & \multicolumn{2}{|c|}{14} & \multicolumn{2}{|c|}{1} & \multicolumn{2}{|c|}{4} & \multicolumn{2}{|c|}{0} & \multicolumn{2}{|c|}{0} & \multicolumn{2}{|c|}{3} & \multicolumn{2}{|c|}{22} \\
\hline \multirow{2}{*}{ Mixto } & 7 & 0 & 1 & 0 & 0 & 0 & 1 & 0 & 0 & 0 & 2 & 0 & 11 & 0 \\
\hline & & & \multicolumn{2}{|c|}{0} & \multicolumn{2}{|c|}{0} & \multicolumn{2}{|c|}{1} & \multicolumn{2}{|c|}{0} & \multicolumn{2}{|c|}{2} & \multicolumn{2}{|c|}{11} \\
\hline \multirow{2}{*}{ A distancia } & 50 & 13 & 4 & 1 & 15 & 1 & 5 & 5 & 0 & 0 & 9 & 7 & 83 & 27 \\
\hline & \multicolumn{2}{|c|}{63} & \multicolumn{2}{|c|}{5} & \multicolumn{2}{|c|}{16} & \multicolumn{2}{|c|}{10} & \multicolumn{2}{|c|}{0} & \multicolumn{2}{|c|}{16} & \multicolumn{2}{|c|}{110} \\
\hline \multirow{2}{*}{ Total } & 68 & 16 & 5 & 2 & 18 & 2 & 6 & 5 & 0 & 0 & 14 & 7 & 111 & 32 \\
\hline & \multicolumn{2}{|c|}{84} & \multicolumn{2}{|c|}{7} & \multicolumn{2}{|c|}{20} & \multicolumn{2}{|c|}{11} & \multicolumn{2}{|c|}{0} & \multicolumn{2}{|c|}{21} & \multicolumn{2}{|c|}{143} \\
\hline
\end{tabular}

Total Modalidad

\begin{tabular}{|c|c|c|c|c|c|c|c|c|c|c|c|c|c|c|}
\hline \multirow{2}{*}{ Presencial } & 130 & 32 & 15 & 14 & 40 & 3 & 14 & 4 & 2 & 3 & 11 & 4 & 212 & 60 \\
\hline & \multicolumn{2}{|c|}{162} & \multicolumn{2}{|c|}{29} & \multicolumn{2}{|c|}{43} & \multicolumn{2}{|c|}{18} & \multicolumn{2}{|c|}{5} & \multicolumn{2}{|c|}{15} & \multicolumn{2}{|c|}{272} \\
\hline \multirow{2}{*}{ Mixto } & 61 & 15 & 10 & 7 & 10 & 1 & 6 & 1 & 0 & 1 & 10 & 3 & 97 & 28 \\
\hline & \multicolumn{2}{|c|}{76} & \multicolumn{2}{|c|}{17} & \multicolumn{2}{|c|}{11} & \multicolumn{2}{|c|}{7} & \multicolumn{2}{|c|}{1} & \multicolumn{2}{|c|}{13} & \multicolumn{2}{|c|}{125} \\
\hline \multirow{2}{*}{ A distancia } & 200 & 50 & 13 & 15 & 50 & 3 & 21 & 9 & 3 & 1 & 41 & 20 & 328 & 98 \\
\hline & \multicolumn{2}{|c|}{250} & \multicolumn{2}{|c|}{28} & \multicolumn{2}{|c|}{53} & \multicolumn{2}{|c|}{30} & \multicolumn{2}{|c|}{4} & \multicolumn{2}{|c|}{61} & \multicolumn{2}{|c|}{426} \\
\hline \multirow{2}{*}{ Gran total } & 391 & 97 & 38 & 36 & 100 & 7 & 41 & 14 & 5 & 5 & 62 & 27 & 637 & 186 \\
\hline & \multicolumn{2}{|c|}{488} & \multicolumn{2}{|c|}{74} & \multicolumn{2}{|c|}{107} & \multicolumn{2}{|c|}{55} & \multicolumn{2}{|c|}{10} & \multicolumn{2}{|c|}{89} & \multicolumn{2}{|c|}{823} \\
\hline
\end{tabular}

car su actividad laboral. El estudio no otorgó incentivos, pero a los participantes se les explicó el beneficio social de su participación en la implementación de las estrategias efectivas para la atención psicológica. Cabe señalar que no hubo conflictos de interés y que el proyecto está avalado por la Facultad de Psicología de la UNAM y la CICAD-OEA, bajo el marco normativo con registro 48850-1666-22-VI-17.

\section{Instrumentos}

Para efectos del estudio, se desarrollaron y validaron instrumentos de evaluación que permiten valorar si el material de capacitación para profesionales de la salud influía favorablemente en la adquisición de competencias profesionales (conocimientos, habilidades y actitudes), para la atención primaria de las adicciones (ver Tabla 2).

Evaluación escrita de conocimientos. Se elaboró un examen de conocimientos con 90 preguntas de opción múltiple con cuatro opciones de respuesta (tres distractores) que evalúa los módulos: 1) conceptos básicos (30 reactivos, por ejemplo: La miosis extrema [puntiforme] es un signo de posible intoxicación/sobredosis por: a. alcohol, b. cocaína, c. opioides, d. solventes); 2) modificación de la conducta de consumo (30 reactivos; por ejemplo: Es un procedimiento en el que se sustituye la respuesta condicional, en el reflejo condicionado de deseo de la droga, por una respuesta más saludable: a. manejo de contingencias, b. condicionamiento, c. contracondicionamiento, d. control de estímulos) y 3) Intervención breve en adicciones (30 reactivos; por ejemplo: Componente de la intervención breve mediante el cual se evalúan las determinantes del consumo en el contexto en el que ocurre: a. análisis funcional, b. control de estímulos, c. manejo de craving, d. solución de problemas). En general, los análisis de confiabilidad y el factorial exploratorio mostraron que el instrumento tuvo un Alfa de Cronbach de 0.96, una varianza explicada del 
Tabla 2

Valores de confiabilidad (Alfa de Cronbach) y validez (Varianza explicada) de los instrumentos de evaluación

\begin{tabular}{lcc}
\hline & $\begin{array}{c}\text { Alfa de Cron- } \\
\text { bach }\end{array}$ & $\begin{array}{c}\text { Varianza } \\
\text { Explicada }\end{array}$ \\
\hline Examen de Conocimientos & 0.96 & 62.57 \\
Módulo Conceptos básicos & 0.82 & 53.19 \\
Módulo Modificación de conducta & 0.91 & 54.35 \\
Módulo Intervenciones breves & 0.97 & 66.34 \\
Evaluación de Habilidades & 0.83 & 57.79 \\
Habilidades & 0.76 & 54.61 \\
Actitudes & 0.75 & 58.32 \\
Evaluación Conductual: IDEARSE & 0.93 & 60.63 \\
Identifica & 0.7 & 77.07 \\
Dialoga & 0.72 & 54.67 \\
Establece & 0.81 & 47.14 \\
Acompaña & 0.85 & 56.35 \\
Retroalimenta & 0.74 & 46.28 \\
Sigue & 0.79 & 61.11 \\
Actitudes & 0.78 & 52.09 \\
Comunicación & 0.69 & 48.37 \\
Empatía & 0.69 & 53.15 \\
Satisfacción & 0.93 & 78.68 \\
\hline & & \\
\hline
\end{tabular}

$63 \%$ y comunalidades mayores a 0.30 en todos los reactivos. Cada factor mostró confiabilidad mayor al 0.82 y varianzas explicadas mayores al 53\% (ver Tabla 2).

Evaluación conductual de habilidades y actitudes. Se generó un sistema de observación directa con dos registros durante un ensayo conductual, aplicando la intervención breve vinculada al ASSIST (The Alcohol, Smoking and Substance Involvement Screening Test). Ambos registros se califican con el puntaje: 0 = "no presenta habilidad/actitud" o 1 = "presenta habilidad/actitud". El primer registro evalúa 29 habilidades, distribuidas en seis factores: Identifica (dos reactivos; por ejemplo, indaga acerca del patrón de consumo); Dialoga (cuatro reactivos; por ejemplo, analiza ventajas del consumo); Establece metas (siete reactivos, por ejemplo: guía a la persona a una meta de reducción o suspensión del consumo o asistencia a tratamiento); Acompaña en la elaboración de planes de acción (siete reactivos, por ejemplo: modela la conducta/habilidad implicada en el plan de acción); Retroalimenta la ejecución (seis reactivos; por ejemplo, realiza escucha reflexiva), y lleva a cabo Seguimiento (cuatro reactivos; por ejemplo: recomienda realizar seguimiento para garantizar que la persona lleve a cabo los planes de acción; el acrónimo final se denomina IDEARSe).

Un análisis de confiabilidad y otro de varianza mostró que el instrumento tuvo un Alfa de Cronbach de 0.93, una varianza explicada del $61 \%$ y comunalidades mayores a 0.30 en todas las habilidades. Cada factor mostró confiabilidad mayor al 0.82 y varianzas explicadas específicas mayores al 46\% (ver Tabla 2). El segundo registro evalúa nueve actitudes a partir de la observación directa, a través de dos escalas: Comunicación (cinco reactivos, por ejemplo: muestra disposición a resolver dudas y aclarar información) y Empatía (cuatro reactivos; por ejemplo: evita la confrontación con la persona). Un análisis de confiabilidad y otro de varianza mostró que el instrumento tuvo un Alfa de Cronbach de 0.70, una varianza explicada del $52 \%$ y comunalidades mayores a 0.30 en todas las actitudes. Cada factor mostró confiabilidad mayor al 0.69 y varianzas explicadas específicas mayores al 48\% (ver Tabla 2).

Finalmente, se diseñó el Cuestionario de Satisfacción constituido por cinco reactivos (por ejemplo: el material que se utilizó y se proporcionó en la capacitación es didáctico) con cinco opciones de respuesta que va desde totalmente en desacuerdo hasta totalmente de acuerdo. El análisis de confiabilidad y otro de varianza mostró que el instrumento tuvo un Alfa de Cronbach de 0.93, una varianza explicada del $79 \%$ y comunalidades mayores a 0.30 en todos los reactivos (ver Tabla 2).

\section{Procedimiento}

El estudio se llevó a cabo a través de un diseño cuasi-experimental post, con grupos de comparación para evaluar conocimientos, habilidades, actitudes (a través de observación directa), así como la satisfacción. Se distribuyó a los participantes de los cinco países entre las tres modalidades de capacitación: presencial, mixta y a distancia. Las capacitaciones tuvieron en común una duración de 40 horas de trabajo, distribuidas a lo largo de tres módulos de contenidos: conceptos básicos, modificación de la conducta de consumo e intervención breve en adicciones.

\section{Evaluación}

Para conocer la adquisición de competencias en función de las modalidades de capacitación, se aplicó la evaluación escrita de conocimientos y la de habilidades, antes y al terminar la capacitación. Ambas evaluaciones se llevaron a cabo durante 60 minutos de trabajo. Posterior a las modalidades de capacitación presencial y mixta, se aplicó la evaluación conductual y el cuestiona- 
rio de satisfacción a lo largo de 60 minutos adicionales de evaluación.

Generalidades de la capacitación presencial, mixta y a distancia. Se elaboraron los manuales de contenidos en extenso, del capacitador, del participante, de diapositivas (todos en formato electrónico [PDF $®]$ ) y el curso en plataforma Moodle ${ }^{\circledR}$, a partir de diversos materiales y contenidos (UTC, mhGAP de OMS y OPS, TreatNet de UNODC, etcétera). El curso promueve una base homologada de información central sobre los problemas por consumo de drogas y el desarrollo de competencias profesionales para abordar elementos biopsicosociales de la dependencia de drogas, como una compleja enfermedad con base cerebral; permite comprender la farmacología de las drogas, el desarrollo de trastornos por uso de drogas, los criterios diagnósticos, la comorbilidad y las necesidades de atención. A través del curso, es posible familiarizarse con la metodología apropiada de intervención a ser utilizada y apoyar el desarrollo de un plan de tratamiento multidisciplinario.

La capacitación se distribuye en tres módulos, a lo largo de los cuales se llevan a cabo las estrategias pedagógicas de aprendizaje: la instrucción verbal de conceptos a través de 221 diapositivas, el modelamiento a través de 88 audiovideos de habilidades y el ensayo conductual a través de 21 viñetas escritas/actividades de aprendizaje; todo ello para la comprensión, el análisis de conceptos y la retroalimentación sobre la ejecución de los profesionales de la salud en formación.

El primer módulo está constituido por 12 horas de capacitación, a través de las cuales se revisan las bases teóricas fundamentales para la atención de las adicciones: definiciones y conceptos, neurobiología de las adicciones, elementos de neuroanatomía y neurofisiología (neuronas, potenciales de acción, neurotransmisión, receptores, neurotransmisores, segundos y terceros mensajeros), mecanismos fisiológicos implicados en la dependencia de las drogas (estudios conductuales en modelos animales y circuito de recompensas), clasificación farmacológica de las drogas (alucinógenos, cannabinoides, depresores, estimulantes, opioides y mezclas), efectos de las drogas (alcohol, cannabis, cocaína, heroína, inhalables y tabaco), modelos y teorías sobre el desarrollo de los trastornos por consumo de drogas (determinantes sociales de la salud, modelo jurídico, de la distribución del consumo, médico tradicional, reducción del daño, privación social, de los factores socioestructurales, de educación para la salud, psicológico individualista, socioecológico, genética y trastornos por consumo de drogas), criterios diagnósticos (CIE-10 y DSM-5), comorbilidad entre el consumo de drogas y los trastornos mentales (ansiedad, depresión, esquizofrenia y suicidio), entre consumo de drogas y enfermedades orgánicas (arritmias cardiacas, cáncer, enfermedad cerebrovascular, hipertensión, lesión renal aguda y patología digestiva), consumo de drogas y enfermedades infecciosas (hepatitis C, tuberculosis y VIH-sida), y consumo de drogas durante el embarazo (síndrome alcohólico fetal, consumo de inhalables durante el embarazo y consumo de cannabis durante el embarazo).

El segundo módulo dura 12 horas y abarca las bases para la práctica e intervención: patrones de consumo (de la experimentación a la dependencia), impacto del consumo de drogas en la salud pública, reducción de la demanda de drogas, el continuo de los servicios de atención, criterios de calidad para centros de tratamiento (recomendaciones generales y criterios indispensables para la apertura y funcionamiento de centros de tratamiento), modificación del consumo de drogas, motivación para cambiar, autoeficacia y modelo trans-teórico del cambio (niveles de cambio, estadios y procesos del cambio).

Para el tercer módulo de 16 horas, se integraron los contenidos de las intervenciones breves recomendadas para favorecer el cambio en el primer nivel de atención y para la referenciación oportuna y efectiva: estrategias motivacionales, detección, tamizaje e intervenciones breves (instrumentos de cribado y tamizaje, intervención breve, sus características, consejo, intervención breve vinculada con la prueba a ASSIST, referencia y contrarreferencia), tratamiento (aspectos generales, mhGAP para superar las brechas en salud mental), principios de la prescripción de farmacoterapia, agentes farmacológicos para el manejo de los trastornos por consumo de drogas (acamprosato, bupropion, disulfiram, metadona, nalmefeno, naloxona, naltrexona, nicotina, topiramato y vareniclina), intervenciones psicosociales avanzadas, principios rectores de las recomendaciones de la OMS para la identificación y manejo del consumo de drogas y los trastornos asociados en mujeres embarazadas.

Particularidades del curso interactivo en plataforma Moodle ®. A partir del desarrollo de los materiales del contenido en extenso, de capacitación, del participante y de las diapositivas, la capacitación en plataforma Moodle ${ }^{\circledR}$, su revisión por pares de expertos de origen de los mismos países y la corrección de estilo de los contenidos, quedó constituida por un total de 312 pantallas a través de 156 ficheros, 74 recursos interactivos (entre ficheros scroll, de pestañas, líneas de tiempo, galerías de imágenes, esquemas de ampliación, acordeones verticales y horizontales, carrusel y animaciones; todo ello a partir de las 221 diapositivas del curso presencial), 79 audios, nueve videos (para los 88 modelamientos), tres evaluaciones finales de conocimientos, cinco autoevaluaciones de ensayo y 21 ejercicios descargables para papel y lápiz. 
Implementación de la capacitación. La logística para las capacitaciones presenciales fue gestionada a través del liderazgo de las Comisiones de Drogas de cinco países, con el apoyo de los Ministerios de Salud y de una universidad pública o privada. A partir de la selección de los participantes por su perfil como personal de salud no especializado en la atención de adicciones y de su designación aleatoria a cada uno de los grupos de capacitación (presencial, mixta o a distancia), se programaron los cursos simultáneos en cada país con una duración total de 40 horas. El rango de trabajo de la fase de capacitación se distribuyó a lo largo de tres meses para los cinco países. En total, se llevaron a cabo 32 cursos de capacitación con un promedio de 25 participantes por grupo.

En cada curso colaboraron entre dos y tres expertos, designados para impartir los tres módulos presenciales, tanto para la modalidad presencial como para la mixta. Se combinaron siempre uno de seis docentes que contaban con grado de Maestría en Psicología de las Adicciones (en la cual se colabora con 2,560 horas de atención de personas con trastornos por consumo de sustancias). Una docente tuvo adicionalmente a la Maestría, el Doctorado en Análisis Experimental del Comportamiento y otro contaba con el Doctorado en Neurociencias. Para las modalidades a distancia y mixta, se designó un asesor experto que fungía como docente de dicho curso a distancia del total de los docentes del equipo. Todos los docentes-asesores expertos se adhirieron a la secuencia y procedimientos de presentación expuesta previamente.

Análisis de los datos. Para el análisis de las evaluaciones, conforme la modalidad de los grupos, se llevaron a cabo análisis multivariados a través del paquete estadístico SPSS ${ }^{\circledR}$, versión 19.

\section{RESULTADOS}

A continuación se presentan los análisis de conocimientos, habilidades, actitudes y satisfacción por cada modalidad de capacitación y país de origen de participantes.

Un análisis multivariado por modalidad de capacitación y rubro de competencias, en todos los países, mostró que, en general, la modalidad de capacitación a distancia generó un incremento significativo en los promedios de conocimientos previos $(M=58.89$; $D E=$ 10.99) a después de la misma $(M=88.30 ; D E=12.35)$ en comparación con la modalidad mixta $(M=58.18 ; D E$ $=19.35$ a $M=77.1 ; D E=16.55)$ y la presencial $(M=$ 37.71; $D E=15.71$ a $M=63 ; D E=15.49 ; F[22,821]=$ 296, $p=.000$ ).

En el caso particular de Argentina, se observó la misma tendencia, es decir, un mayor incremento en la ca- pacitación de modalidad a distancia (de $M=58.78$; $D E=11.00$ a $M=85.1 ; D E=12.08)$ que en la mixta $(M$ = 69.44; $D E=15.91$ a $M=72 ; D E=21.40)$ y presencial $(M=38.47 ; D E=17.64$ a $M=64.7 ; D E=13.00 ; F[22$, $135]=70.73, p=.000)$. En Colombia la capacitación a distancia, también, generó el promedio de conocimientos más elevados $(M=48.89 ; D E=13.81$ a $M=90 ; D E$ = 9.53), seguida por la mixta $(M=61.11 ; D E=14.13 \mathrm{a}$ $M=78.8 ; D E=11.77)$ y la presencial $(M=51.89 ; D E=$ 12.51 a $M=64.9 ; D E=11.92 ; F[22,57]=94.67, p=$ $.000)$. En el caso de El Salvador, de la misma manera, la capacitación a distancia generó el promedio de conocimientos más elevados $(M=66.67 ; D E=19.51$ a $M=79$; $D E=18.88)$, seguida por la mixta $(M=60.64 ; D E=15.49$ a $M=70.5 ; D E=12.92)$ y la presencial $(M=31.02 ; D E$ $=13.58$ a $M=61.7 ; D E=17.77 ; F[22,84]=119.2, p=$ .000). En México la capacitación a distancia, también, generó el promedio de conocimientos más elevados ( $M$ $=43.17 ; D E=19.75$ a $M=92.8 ; D E=8.77$ ), seguida por la mixta $(M=59.42 ; D E=19.70$ a $M=82.6 ; D E=13.32)$ y la presencial $(M=38.1 ; D E=16.22$ a $M=66.6 ; D E=$ 15.13; $F[22,396]=383.37, p=.000)$. Finalmente, para Uruguay la tendencia fue la misma. La capacitación a distancia también generó el promedio de conocimientos más elevados $(M=30.00 ; D E=9.79$ a $M=85.30 ; D E=$ 14.31), seguida por la mixta $(M=35.19 ; D E=6.56$ a $M$ $=54.00 ; D E=13.99)$ y la presencial $(M=34.74 ; D E=$ 10.57 a $M=50.00 ; D E=10.83 ; F[22,141]=106.30, p$ $=.000$ ).

En la Figura 1 se observan los porcentajes promedio por cada uno de los tres módulos de conocimientos en cada modalidad, por cada país de origen de los participantes (gráficas de la primera columna izquierda de la Figura 1). Por lo tanto, aunque se observa un incremento de conocimientos, el mayor impacto se registra en la modalidad a distancia, con la plataforma Moodle $\AA$, que tiene un bajo costo de operación, excepto para los participantes de Uruguay en el módulo de conceptos básicos.

En cuanto a las habilidades para la intervención breve (Indagar, Dialogar, Establecer metas, Acompañar en la elaboración de planes de acción, Retroalimentar a las personas atendidas y Seguimiento de los logros; IDEARSe), el análisis multivariado indicó que no hubo diferencias significativas en los porcentajes promedio entre las modalidades de capacitación presencial $(M=74.21 ; D E$ $=26.77)$ y mixta $(M=66.63 ; D E=24.94 ; F[22,821]=$ $3.45, p=.065)$. Lo que sí se observa es una distinción entre las habilidades con un mayor porcentaje promedio y las de menor porcentaje. Es decir, las habilidades de mayor porcentaje promedio fueron: Indagar, en las modalidades de capacitación presencial $(M=94.79$; $D E=19.12)$ y mixta $(M=95.07 ; D E=19.19)$; seguidas por Dialogar, para presencial $(M=79.01 ; D E=31.64)$ y 


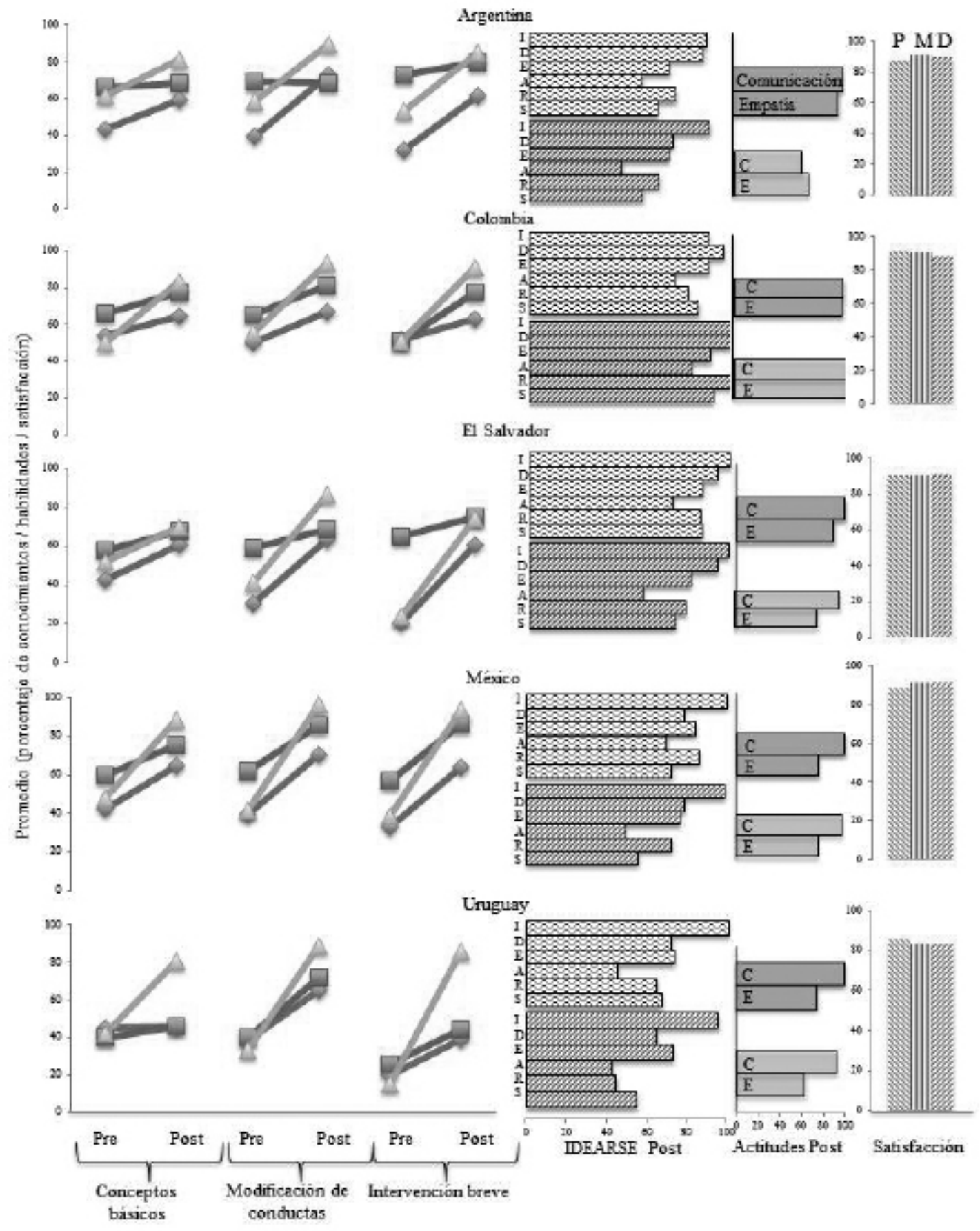

\section{Nota $\odot$ Presencial $a$ Mixto $A$ A distancia}

Figura 1. Porcentajes promedio en cada módulo de conocimientos: conceptos básicos, modificación de conductas, intervención breve, antes y después de cada modalidad de capacitación (gráficas primera columna laterales izquierdas), habilidades de consejo breve (IDEARSe; gráficas de la segunda columna), actitudes (comunicación y empatía; gráficas de la tercera columna) y satisfacción general (gráficas de la cuarta y última columna), al finalizar las mismas modalidades de capacitación, para todos los participantes por país de origen. 
mixta $(M=77.17 ; D E=25.99)$; Establecer metas, para presencial $(M=78.41 ; D E=28.99)$ y mixta $(M=74.58$; $D E=25.15) ;$ Retroalimentar, presencial $(M=76.65 ; D E$ = 33.12) y mixta $(M=68.12 ; D E=31.54)$; Seguimiento, presencial $(M=71.33 ; D E=34.35)$ y mixta $(M=58.48$; $D E=39.29)$; y Acompañar en la elaboración de planes de acción, para presencial $(M=61.25 ; D E=39.16) \mathrm{y}$ mixta $(M=49.40 ; D E=39.73)$. Es decir, como se muestra en la segunda columna de gráficas de la Figura 1, la habilidad con los porcentajes promedios más altos fue Indagar, y la de los más bajos Acompañar, para todos los participantes por país de origen (excepto presencial, en Colombia).

Con respecto a las actitudes, el análisis multivariado indicó que, después de la capacitación, los porcentajes promedio de actitudes fueron similares para las modalidades mixta $(M=95.37 ; D E=13.80)$ y a distancia $(M=95.74 ; D E=12.65 ; F[5,821]=0.01, p=.937) . \mathrm{El}$ porcentaje promedio de comunicación $(M=96.52$; $D E=$ 10.62) fue más elevado que el de empatía $(M=77.04$; $D E=33.13)$, tanto en la capacitación presencial como a distancia (para comunicación $M=96.74 ; D E=11.92$ y para empatía $M=74.64 ; D E=32,67$ ). Como se observa en las gráficas de la tercera columna de la Figura 1, las tendencias en todos los países fueron similares, excepto para Argentina en la modalidad mixta y para Colombia, donde ambas modalidades mostraron el mayor porcentaje posible.

Finalmente, con relación a los niveles de satisfacción de los profesionales con el curso, el análisis multivariado indicó que no hubo diferencias significativas entre los porcentajes promedio de satisfacción por modalidad de capacitación: presencial $(M=89.57 ; D E=19.53)$, mixta $(M=91 ; D E=13.33)$ y a distancia $(M=88.44 ; D E=$ $12.71 ; F[2,821]=0.90, p=.409)$. Es decir, como se observa en las gráficas de la cuarta y última columna de la Figura 1, todos los participantes reportaron porcentajes de satisfacción elevados. El país con el mayor porcentaje promedio de satisfacción fue Argentina para la modalidad mixta $(M=92.00 ; D E=16.55)$, y el más bajo Uruguay para la misma modalidad $(M=82.96$; $D E$ $=14.68)$, pero sin diferencias significativas.

\section{DISCUSIÓN}

El objetivo del trabajo consistió en evaluar el nivel de conocimientos, habilidades y actitudes de los profesionales de la salud para la intervención breve en adicciones, en función de la modalidad de capacitación: presencial, mixta y a distancia, como estrategia de validación de los materiales didácticos del currículum universal de tratamiento, en su versión para profesionales de la salud. La capacitación en sus tres modalidades se asoció con la adquisición de los conocimientos sobre los conceptos básicos en materia de adicciones, los mecanismos motivacionales del cambio y las estrategias de intervención breve para atender a las personas que consumen sustancias. En ese sentido, en general se observó que los materiales diseñados, revisados en estilo, estrategia pedagógica, diseño instruccional, programación de recursos didácticos e integración de los mismos, en plataforma Moodle ${ }^{\circledR}$, resultaron en un paquete de herramientas tecnológicas a través de las cuales y con un curso de 40 horas de implementación en sus tres modalidades (presencial, mixta y a distancia), permitieron el incremento de conocimientos y trabajar habilidades y actitudes, conforme a la evaluación escrita y a través de la observación directa de su ejecución, tal como lo recomendaron Abd-Hamid y Walkner (2017).

Los hallazgos concuerdan con lo reportado por Morales-Chainé et al. (2013), quienes demostraron que los profesionales de la salud no solamente adquieren conocimientos a través de capacitaciones auxiliadas con herramientas didácticas en materia de adicciones, sino que es posible observar la ejecución de las habilidades para la intervención breve. La diferencia es que en el actual estudio fue posible determinar la ocurrencia de todas las conductas asociadas con esta estrategia, incluyendo la capacidad de retroalimentar a las personas que consumen sustancias y planear el seguimiento de los logros alcanzados, que no se observó en el trabajo previamente citado.

La innovación del estudio actual toma la dirección de las aportaciones previas de capacitación en plataforma Moodle ${ }^{\circledR}$ de Vargas et al. (2012) y Barrera et al. (2017). Es decir, con la construcción de una herramienta basada en las tecnologías de la información y comunicación (TIC) como los ficheros scroll, de pestañas, líneas de tiempo, galerías de imágenes, esquemas de ampliación, acordeones verticales y horizontales, carrusel, animaciones, audios, videos de modelamiento y autoevaluaciones de ensayo, es posible incrementar la eficiencia de una estrategia de capacitación para aquellas comunidades virtuales dispuestas a adoptar la dinámica de trabajo. Los hallazgos señalan la posibilidad de aprender por medio de las TIC conceptos básicos sobre neurobiología, neuroanatomía, neurofisiología (de la dependencia de las drogas), clasificación farmacológica, efectos de las drogas, modelos y teorías sobre el desarrollo de los trastornos por consumo de drogas, criterios diagnósticos y comorbilidad física y mental. Adicionalmente, parecen favorecer el entrenamiento de las habilidades y la medición de las actitudes para la práctica de las intervenciones breves recomendadas para promover el cambio en el primer nivel de atención. La estrategia actual constituye, por lo tanto, una herramienta de disemina- 
ción exitosa, basada en los contenidos recomendados por los organismos internacionales, pero validados, por su derivación de la evidencia empírica, en escenarios controlados; ahora directamente en el campo de aplicación clínica de los principios básicos del comportamiento (Morales-Chainé, 2012).

Tales hallazgos se observan en los profesionales de tres de los cinco países con los que se trabajó. Es decir, el nivel de generalidad se distribuye en los casos particulares de los participantes de Colombia, El Salvador y México, donde se encontró que las tres modalidades de capacitación, y primordialmente a distancia (con gran realce en México), permitieron fortalecer sus conocimientos relativos a los conceptos básicos, la modificación de conducta e intervención breve en el primer nivel de atención en adicciones. A través de la observación directa, también fue posible sugerir que los niveles de dominio de la intervención breve (IDEARSe) por los participantes de los tres países fueron habilidades y actitudes producto de las capacitaciones presencial y mixta. Solamente debe tomarse con reserva el caso particular de los participantes originarios de México, donde la modalidad de capacitación mixta específicamente en la capacidad de guiar la elaboración de planes de acción, durante la intervención breve, parece un tanto más limitada. No obstante, en el caso de las actitudes medidas también a través de observación directa, los niveles de comunicación y empatía fueron elevados en los profesionales.

En el ámbito de la diseminación de las estrategias de intervención basadas en la evidencia (Morales-Chainé, 2012) resulta fundamental también analizar los resultados de menor alcance como los observados en Argentina y Uruguay. En el caso particular de Argentina, se encontró que la capacitación presencial y, primordialmente a distancia, permitieron fortalecer los conocimientos relativos a los conceptos básicos, la modificación de conducta e intervención breve de los profesionales de la salud. Sin embargo, la modalidad mixta solamente favoreció el incremento en los conocimientos para la intervención breve. Los niveles más reducidos en esta modalidad se observaron en la capacidad para guiar la elaboración de planes de acción, el seguimiento y los niveles de comunicación. En el caso de Uruguay, se observó que la modalidad de capacitación a distancia permitió mejorar los conocimientos relativos a los conceptos básicos, la modificación de conducta e intervención breve. Sin embargo, en las modalidades mixta y presencial, el conocimiento no alcanzó los niveles aprobatorios. Los niveles más reducidos se observaron para la capacidad de guiar la elaboración de planes de acción (en la modalidad presencial), de retroalimentación y seguimiento (en la modalidad mixta), durante la intervención breve.
En vías de una posible explicación, cabe considerar un estudio reciente en el que Martínez, Jiménez, Félix y Morales-Chainé (2018) analizaron las barreras de la transferencia de intervenciones breves en adicciones, en el primer nivel de atención, con 756 profesionales de la salud. Los autores indicaron que los participantes reportaron, dentro de las barreras, la dificultad con los tópicos, con el marco conceptual que los sustenta, pero particularmente las dificultades que perciben para guiar a las personas atendidas dentro de la implementación de las intervenciones breves. Los autores consideran como crucial la inclusión de la supervisión para que los profesionales logren identificar y resolver tales dificultades, así como los logros y avances durante la implementación de las intervenciones breves; dicho ejercicio podría garantizar su adopción exitosa. Entonces, una investigación adicional deberá señalar si el monitoreo de las estrategias de modelamiento, ensayo y retroalimentación para los módulos de conceptos básicos y modificación de conducta en la modalidad mixta, para los profesionales de la salud originarios de México, Colombia, el Salvador, pero sobre todo de Argentina y Uruguay, promoverían el dominio de los conocimientos señalados en beneficio de aquellas poblaciones atendidas.

En concreto, en el presente estudio y para la estrategia de intervención breve, que promueve una reducción en el consumo de alcohol en adolescentes (Vargas et al., 2012) y adultos (Barrera et al., 2017), fue posible generar y medir un conjunto de competencias a partir de diversas modalidades de diseminación (Abd-Hamid \& Walkner, 2017). De tal manera que, en formato presencial, tal como Morales-Chainé et al. (2013) lo habían demostrado, y en modalidad a distancia, como Barrera et al. (2017) lo sugirieron, los profesionales de la salud no sólo adquirieron conocimientos, habilidades y actitudes, sino que reportaron altos índices de satisfacción, con los contenidos, materiales, conocimientos y habilidades adquiridas.

Por lo tanto, el uso de las TIC favorece ampliamente la adquisición de conocimientos con plena calidad en la formación, comparado con el uso de las tecnologías tradicionales (Abd-Hamid \& Walkner, 2017), es decir, contra las estrategias presenciales. A pesar de los incrementos significativos en conocimientos, es necesario señalar que se observó un menor dominio en los conceptos básicos sobre epidemiología, neurobiología, farmacología, criterios diagnósticos y comorbilidad psiquiátrica, después de la capacitación presencial. No obstante, se observó un mayor dominio de conocimientos con relación a los niveles, estadios y procesos de cambio, detección, tamizaje, intervenciones breves y tratamiento en adicciones. A través del sistema de evaluación conductual, en el estudio actual también fue posible evaluar 
las habilidades para la intervención breve: indagar, dialogar, establecimiento de metas, acompañar en la elaboración de planes de acción, retroalimentar la ejecución y planear el seguimiento (Morales-Chainé et al., 2013; Morales-Chainé et al., 2016), como resultado de la capacitación de 40 horas en modalidades presencial, mixta y a distancia. También, a través del sistema de evaluación conductual fue posible evaluar las actitudes positivas de la implementación de la intervención breve: empatía y comunicación. La evaluación basada en situaciones simuladas o que estimulan la aplicación del conocimiento parecen permitir al investigador concluir más allá de la adquisición de conceptos, en el aprendizaje de habilidades y actitudes que incrementan la probabilidad de que los profesionales de la salud utilicen tales competencias en el escenario real con las personas que atienden en el ámbito clínico y de primer nivel de atención (Abd-Hamid \& Walkner, 2017).

En este sentido, en un estudio reciente, Félix, Morales-Chainé y Santoyo (2019) evaluaron cómo el nivel de implementación de los procedimientos conductuales de las intervenciones breves por 109 profesionales de la salud favoreció la conclusión exitosa de la intervención para 270 personas atendidas en escenarios reales. Sus resultados indicaron que, específicamente, los procedimientos de establecimiento de metas, planeación de conducta alternativa, y particularmente los ensayos conductuales entre el profesional y las personas atendidas predijeron la conclusión exitosa de la intervención. Si en el actual estudio los profesionales de la salud mostraron incrementos en las habilidades y actitudes relativas a la intervención breve (IDEARSe), los cursos de acción alternativos deberán dirigirse a medir el nivel de implementación y supervisar a los profesionales de la salud en binomio con las personas atendidas en escenarios reales, como Félix et al. (2019), y observar los alcances de la capacitación del UTC para profesionales de la salud.

Así, los materiales desarrollados promovieron la adquisición de competencias profesionales: conocimientos, habilidades y actitudes, en el ámbito de la atención primaria de las adicciones por profesionales de la salud, no especializados; y la evaluación mediante observación directa permitió corroborar que el material del UTC para profesionales de la salud favorece dicha adquisición (Abd-Hamid \& Walkner, 2017), específicamente en la implementación de estrategias clave como la detección oportuna, la intervención breve y el manejo inicial de casos. Los hallazgos del presente estudio podrían constituir evidencia inicial de que, dado que los materiales en plataforma Moodle $®$ otorgaban retroalimentación inmediata de las acciones emitidas en los ejercicios y recursos programados, esta condición favorece un aprendizaje significativo de mayor permanencia (Thalheimer,
2008). Investigación adicional deberá corroborar dicha premisa.

Conforme sugirieron Morales-Chainé et al. (2016), el presente estudio permite ilustrar el nivel de conocimientos teóricos y prácticos adquiridos, el efecto de la capacitación de los profesionales de la salud y el aporte práctico de las tecnologías de la información en el entrenamiento para la atención de las adicciones. En prospectiva, procede evaluar el nivel de implementación de las competencias en la atención directa de las personas que consumen sustancias psicoactivas y la reducción o eliminación de su conducta de consumo de sustancias, como lo reportaron Barrera et al. (2017) y Félix et al. (2019). Estudios futuros deberán considerar evaluaciones de proceso que permitan explicar cómo las TIC promueven la adquisición de habilidades y actitudes, e incrementan el sentido de involucramiento y motivación durante el aprendizaje a distancia (Malamed, 2015).

Para el desarrollo de un proceso de garantía de calidad en esta materia, es esencial que en cada país se propicie la participación conjunta de las autoridades responsables de atender la problemática de drogas, de diferentes áreas del sector salud con quienes proveen servicios de atención a las personas con problemas por consumo de alcohol, tabaco y otras drogas.

\section{FUENTES DE FINANCIAMIENTO}

El presente trabajo se realizó con apoyo financiero del Convenio de Colaboración con la Secretaría General de la Organización de los Estados Americanos, por medio de la Secretaría Ejecutiva de la Comisión Interamericana para el Control del Abuso de Drogas y de la Facultad de Psicología de la UNAM, registro 48850-1666-22-VI17 a la responsable del proyecto, doctora Silvia Morales Chainé.

\section{CONFLICTO DE INTERÉS}

Los autores declaran no tener conflictos de interés.

\section{REFERENCIAS}

Abd-Hamid, N. H., \& Walkner, R. N. L. (2017). Evidence-Based Best Practices in Designing and Developing Quality eLearning for the Public Health and Health Care Workforce. Pedagogy in Health Promotion: The scholarship of Teaching and Learning, 3(1S), 35S-39S. doi: 10.1177/2373379917692818

Atlas de Salud Mental. (2014) Recuperado de: http://www.who.int/ mental_health/evidence/atlas/mental_health_atlas_2014/es/.

Barrera, R. C., Echeverría S. V. L., \& Morales-Chainé, S. (2017). Educación en línea: Resultados de la capacitación a profesionales de la salud en una intervención breve para bebedores 
excesivos. Revista Electrónica de Psicología Iztacala, 20(4), 1552-1570

Comisión Interamericana para el Control del Abuso de Drogas. (2017). Recuperado de http://www.cicad.oas.org/Main/Template.asp?File=/reduccion_demanda/proccer/proccer_spa.asp.

Estrategia Hemisférica sobre Drogas. (2010). Recuperado de http:// www.cicad.oas.org/apps/Document.aspx?ld=954.

Félix, R. V., Morales-Chainé, S., \& Santoyo, V. C. (2019). Implementación de procedimientos conductuales: Efectos en alta post tratamiento del usuario de drogas. Health and Addictions. Salud y Drogas, 19 (1), 127-137).

Informe Mundial de Drogas. (2018). Recuperado de https://www. unodc.org/wdr2018/prelaunch/WDR18_ExSum_Spanish.pdf

Malamed, C. (2015). Visual design solutions: Principles and creative inspiration for learning professionals. Hoboken, NJ: Wiley.

Martínez, M. K. I., Jiménez, P. A. L., Félix, R. V., \& Morales-Chainé, S. (2018). Impact of a brief intervention programs in clinical practice: Barriers and adaptations. International Journal of Psychological Research, 11(2), 27-34. doi:10.21500/20112084.3485

Morales-Chainé, S. (2012). Las habilidades metodológicas y conceptuales en el quehacer cotidiano de la ciencia del comportamiento. Revista Mexicana de Investigación en Psicología, 4(2), 126-129.

Morales-Chainé, S., Martínez-Martínez, K. I., Carrazcosa-Venegas, C., Chaparro-Caso López, A. A., \& Martínez-Ruiz, M. J. (2013). Evaluación de habilidades de consejo breve en el ámbito de las adicciones. Salud y Drogas, 13(2), 109-116.

Morales-Chainé, S., Robles, S. N., Nieto, J., \& Martín del Campo, S. R. (2016). Diseminación de las intervenciones breves en Méxi- co. Resumen del informe presentado en la reunión de expertos para la reducción de la demanda, CICAD. Inebria Latina, 2(1), 3-5.

Organización de los Estados Americanos. (2013). El problema de las Drogas en las Américas; Estudios: Drogas y Salud Pública. Washington, DC: OEA.

Organización Mundial de la Salud. (2004). Recuperado de http:// www.who.int/topics/es/

Rodríguez, C., Echeverría, V., Martínez, K., \& Morales-Chainé, S. (2017). Intervención breve para adolescentes que inician el consumo abusivo de alcohol y otras drogas: su eficacia en un escenario clínico. Revista Internacional de Investigación en Adicciones, 3(1), 16-27. doi: 10.28931/riiad.2017.1.03

Plan de Acción Hemisférico sobre Drogas. (2016-2020). Recuperado de http://www.cicad.oas.org/mem/Activities/PoA/PoA-Version_ Final-ESP.pdf.

Thalheimer, W. (2008). Providing learners with feedback-Part 2 : Peer-reviewed research compiled fo training education, and e-learning. Retrieved from http://willthalmeimer.typepad.com/ files/providing_learners_with_feedback_part2_may2008.pdf

Vargas, E., Martínez-Martínez, K. I., Pedroza-Cabrera, F. J., \& Morales-Chainé, S. (2012). Incursión del Programa de Intervención Breve para Adolescentes en el E-Learning: Resultados del piloteo. Investigación y Ciencia de la Universidad Autónoma de Aguascalientes, 55, 42-47.

Zepeda, H. R., Ávila, S. R., Morales-Chainé, S., \& Martín del Campo, S. R. (2017). Características Sociodemográficas en la deserción de las intervenciones breves contra las adicciones. Ciencias, 122-123, 134-145. 